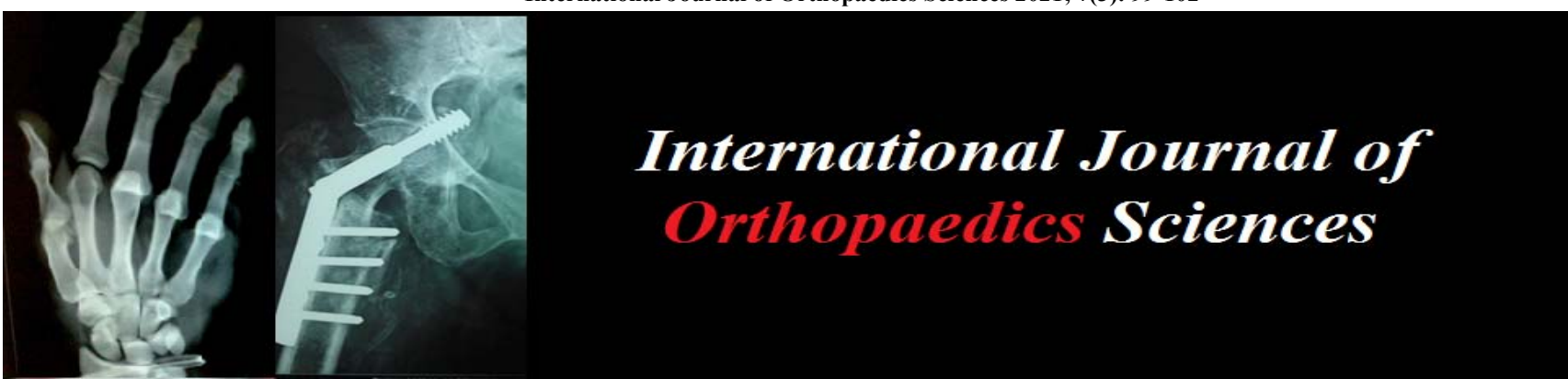

E-ISSN: 2395-1958

P-ISSN: 2706-6630

IJOS 2021; 7(3): 99-102

(C) 2021 IJOS

www.orthopaper.com

Received: 17-04-2021

Accepted: 23-06-2021

Dr. Sunil Gottipati

MS(Ortho), Assistant Professor,

Department of Orthopaedics,

Gitam Institute of Medical

Sciences and Research (GIMSR)

Visakhapatnam, Andhra

Pradesh, India

Dr. Y Siva Sai Kumar Reddy MS(Ortho), Assistant Professor, Department of Orthopaedics, Gitam Institute of Medical Sciences and Research (GIMSR) Visakhapatnam, Andhra Pradesh, India
Corresponding Author: Dr. Y Siva Sai Kumar Reddy MS(Ortho), Assistant Professor, Department of Orthopaedics, Gitam Institute of Medical Sciences and Research (GIMSR), Visakhapatnam, Andhra Pradesh, India

\section{Antiglide plating for distal fibula fractures caused by supination-external rotation injury}

\author{
Dr. Sunil Gottipati and Dr. Y Siva Sai Kumar Reddy
}

DOI: https://doi.org/10.22271/ortho.2021.v7.i3b.2734

\section{Abstract}

Supination-external rotation type of fracture is the most common pattern of ankle fractures. Anatomical reduction of ankle is always desired and poor reduction usually leads to shortening of fibula, or alter the biomechanics of ankle leading to chronic pain as a result of failure of fixation always. Both lateral and antiglide plating of fibula achieve in accurate anatomical reduction of these fractures. But, maintaining the reduction until bony union is also required to avoid failure of hardware or non union. Antiglide construct enhances biomechanics strength of the fixation device due to its anti gliding effect of the applied plate upon weight bearing and thereby is more favourable. This study evaluated clinical outcomes and complications seen in fixations done by antiglide technique done during 2018-2021 among patients admitted with SER pattern injuries at our institute. A total of 24 patients were included in the study. Mean operative time in the study group was $57.5 \pm 27.4$ (range 20-120) minutes. The most commonly used plates were five-hole plates in $10(42 \%)$ patients, six holed plates in $10(42 \%)$ patients and seven holed in $4(16 \%)$ patients. The median number of screws distal to the fracture line in the antiglide plate was 0.5 (range $0-2) ; 10(50 \%)$ fibulas with no fixation, $8(40 \%)$ with one and $2(10 \%)$ with two screws. The AOFAS in the antiglide group performed at one year after surgery was $94.5 \pm 6.0$ (range 85-100). We conclude that the use of the antiglide plate in a posterolateral position, without using unreasonable distal screws, is an appropriate method of fixation in every short oblique lateral malleolus fractures occurring as a part of SER pattern injury of ankle.

Keywords: Antiglide plating, distal fibula fracture, SER injury Ankle, Antiglide principle for SER injuries of Ankle

\section{Introduction}

The need for anatomical reduction of distal fibula fractures in ankle injuries is emphasised. In such displaced fractures, anatomical length and rotational restoration of the distal fibula is required, usually by open reduction, lag screw insertion and plate fixation ${ }^{[1]}$. Supinationexternal rotation type of fracture is the most common pattern of ankle fractures. The first structure to rupture is the anterior tibiofibular ligament; however, it is discussed ${ }^{[2]}$. The second structure is fibula fracture followed by posterior malleolus fracture in the third stage and the fourth stage with deltoid ligament rupture or medial malleolus fracture ${ }^{[3]}$ Treatment of these fractures mainly depends on its displacement. While a number of methods of fixation like intra medullary by Rush nail and longer screws, and plating methods like neutralisation, compression, locking plates are available, the lateral plating as advocated by AO principles is widely followed ${ }^{[4]}$. Limitations of lateral plating include, hardware prominence, penetration of screws in the the ankle joint and syndesmosis and often requiring only unicortical purchase to avoid such joint penetrations making the construct less stable. Whatever is the fixation of choice, anatomical reduction of fracture with ankle stability is required ultimately to maintain length of fibula and restore normal biomechanics of ankle. Brunner and Weber described the method of posterior antiglide plating $[5,6]$. The antiglide plate prevents the distal oblique fragment from gliding through the proximal fragment when axial forces are applied. The limitations of lateral plating like hardware prominence and screw penetration in to the ankle joint are also avoided by this technique of posterior antiglare plating. Although, there are some studies which have shown many advantages of posterior plating over lateral, there are certain apprehensions with respect to complications like peroneal tendinopathy reported in some 
papers. The purpose of this study is to evaluate clinical outcomes and focus on complications seen in fixations done by antiglide technique.

\section{Materials and Methods}

Between March 2018 and June 2021, we prospectively identified and treated patients with lateral malleolus fractures with Danis-Weber B type. The fractures had to be closed and caused by a supination-external rotation mechanism according to Lauge-Hansen classification. The fractures could be in the second (isolated lateral malleolus fracture), the third (lateral malleolus and posterolateral tibia fracture) or in the fourth (lateral malleolus, posterolateral tibia, and medial malleolus fracture) stage of a supination-external rotation injury. Patients younger than 18 years of age, polytraumatized, with additional lower limb injury, old and pathological fractures were excluded. We also excluded patients who did not attend the scheduled controls. Finally, we have evaluated 24 patients with a minimum of one-year follow-up. Basic demographic data (age, gender), time data (operative time, duration of hospitalisation) were recorded. All the fibula fixations in the study group were fixed by anti glide plating using semitubular plate. Torniquet was used for the surgical procedure. All patients had their surgery within 14 days which was depended by soft tissue conditions. Antiglide plating works on the principle that the one-third tubular plate, which is placed posterolaterally on the fibula in an anti-glide position, indirectly reduces the fracture and acts as a buttress to resist the posterior and proximal displacement of the distal fragment [Fig 1]. Additionally, when the fracture configuration allows, the fracture can be compressed with a lag screw, inserted through the plate. A posterolateral incision given over fibula and a careful soft tissue dissection after cutting fascia is done to expose the fracture and to access posterior aspect of fibula. Preliminary reduction is done by gentle axial traction and rotation of foot, and at times if required a 2 point reduction forceps is used to reduce the fracture. Accurate positioning of the plate is essential in obtaining the buttress effect and allowing the insertion of a lag screw. The proximal hole closest to the fracture site should be located 3-5 mm proximal to the fracture line. To improve the anti-glide buttressing effect, the plate is slightly bent at its distal end. The central section of the plate is left straight so that when the central screw is tightened, it will pull the plate against the bone. This applies posterior compression on the distal fragment, which augments compression across the fracture plane. Once the plate position is done, the apex screw in proximal fragment closest to fracture line is inserted and compression of the fracture due to buttressing affect can be noted. After fixation of other relevant injuries, wound is washed and closed in layers. [Fig 2]

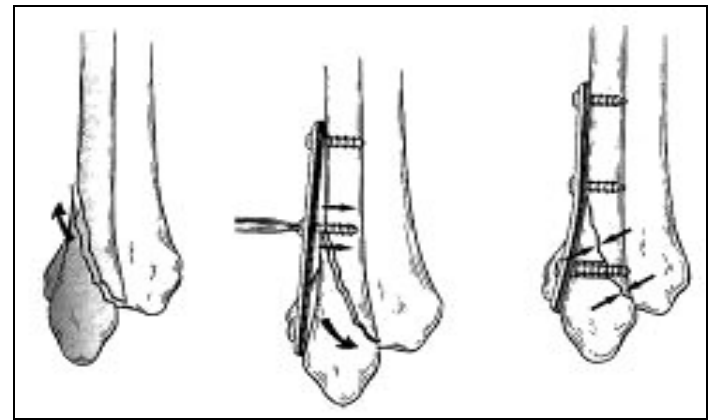

Fig 1: Principle of Antiglide plating. Position of apex screw and reduction of fracture by buttressing effect.

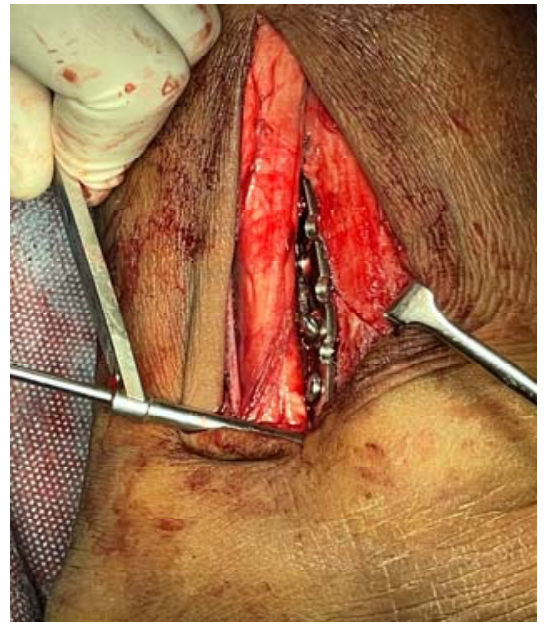

Fig 2: Intra operative image showing posterior antiglide plating of a oblique distal fibula fracture reduced with buttress effect.

Postoperatively, the ankle was immobilised for a maximum of 3 weeks only if the fracture of posterior malleolus was present. Passive motion in ankle joint was allowed on the second postoperative day with the active motion after three weeks. Partial weight bearing was allowed three weeks after surgery with full weight bearing after six weeks. Radiological evaluation was performed at six weeks, three months, six months and one year after surgery. Functional assessment according to the American Orthopaedic Foot and Ankle Society hind foot-ankle score (AOFAS) were performed one year after surgery. All complications were recorded. Peroneal tendinopathy was diagnosed when there was a pain to palpation directly on the peroneal tendons and if pain presented in foot inversion and dorsal flection. If patients showed no pain but only some tenderness over the plate, this was recorded as hardware irritation.

\section{Results}

A total of 24 ankle fractures with distal fibula fractures caused by Supination external rotation injury fixed by posterior anti glide plating of fibula was evaluated in this study. According to Lauge-Hansen classification of supination-external rotation injury, a total of $14(60 \%)$ patients was classified as stage 2,1 (4\%) patients as stage 3 and $9(36 \%)$ patients with stage 4. The posterolateral tibia fracture was treated only in one case by anteroposterior cancellous bone screw fixation. The medial malleolus fractures were fixed by cancellous bone screw (10 cases) or by two Kirschner wire and figure of eight cerclage (4 cases) and in one instance without any fixation. There were $13(54.2 \%)$ male and $11(45.8 \%)$ female. The average patient age was $38.6 \pm 15.4$ (range 23-68) years. Mean operative time in the study group was $57.5 \pm 27.4$ (range 20-120) minutes. The most commonly used plates were five-hole plates in $10(42 \%)$ patients, six holed plates in $10(42 \%)$ patients and seven holed in $4(16 \%)$ patients. The median number of screws distal to the fracture line in the antiglide plate was 0.5 (range $0-2) ; 10(50 \%)$ fibulas with no fixation, $8(40 \%)$ with one and $2(10 \%)$ with two screws. The AOFAS in the antiglide group performed at one year after surgery was $94.5 \pm 6.0$ (range 85-100). Complications observed in patients of an antiglide plate is tabulated [Table 1]. There was no case of tendinopathy. Hardware removal was undertaken in $4(16.6 \%)$ patient in antiglide group. One patient with superficial infection treated with negative wound pressure therapy. 
Table 1: Complications in the Antiglide plating of fibula study group.

\begin{tabular}{|c|c|c|}
\hline Complication & $\begin{array}{c}\text { No Of } \\
\text { Patients }\end{array}$ & Percentage \\
\hline Superficial Infection & 1 & $4 \%$ \\
\hline Deep Infection requiring & Nill & N/A \\
\hline $\begin{array}{c}\text { Hardware irritation requiring } \\
\text { removal }\end{array}$ & 4 & $16.6 \%$ \\
\hline Peroneal tendinopathy & Nill & N/A \\
\hline Non union & Nill & N/A \\
\hline Malunion & Nill & N/A \\
\hline DVT & 1 & $4 \%$ \\
\hline
\end{tabular}

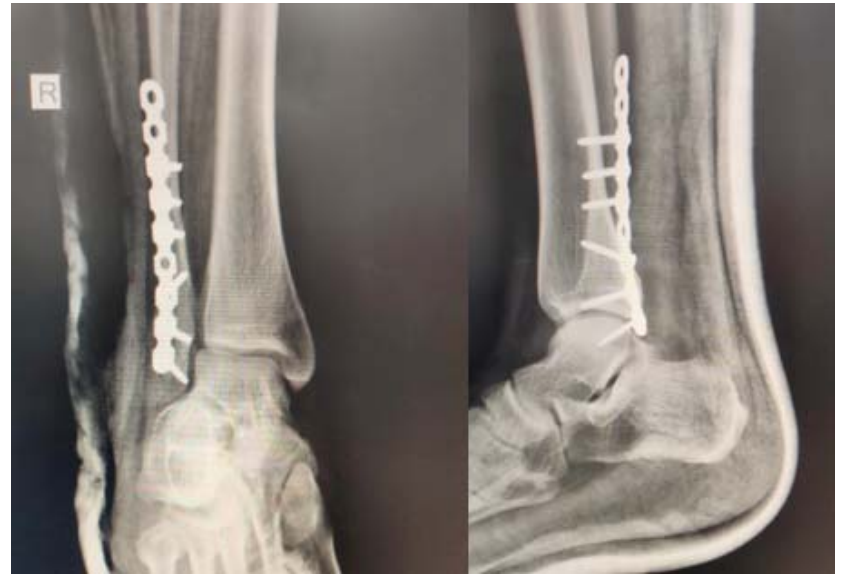

Fig 3: Post op $\mathrm{X}$ ray of isolated fibula fracture fixed with posterior antiglide plating.

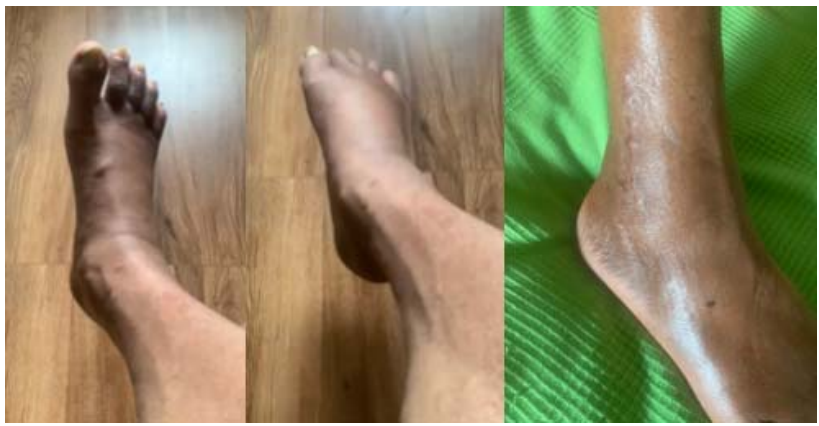

Fig 4: Functional outcome showing good ROM of ankle and healed surgical scar.

\section{Discussion}

Anatomical reduction of ankle is always desired and poor reduction usually leads to shortening of fibula, or alter the biomechanics of ankle leading to chronic pain as a result of failure of fixation always. Both lateral and antiglide plating of fibula achieve in accurate anatomical reduction of these fractures. But, maintaining the reduction until bony union is also required to avoid failure of hardware or non union. Antiglide construct enhances biomechanics strength of the fixation device due to its anti gliding effect of the applied plate upon weight bearing and thereby is more favourable. In biomechanics comparison studies evaluating antiglide plating and lateral plating, antiglide plating was found to be superior in healthier bones. Additional lag screw definitely adds to its biomechanics strength compared to lateral plating couterparts. [7-9]

Since 1982, when Brunner and Weber described antiglide plate technique, many authors encourage and recommended this fixation. ${ }^{[10-11]}$ Distal fibula fragment in the SER Weber B type fracture has a typical posterolateral displacement usually with slight shortening ${ }^{[12]}$. Placing a plate in antiglide fashion automatically reduces the distal fragment by screwing the plate to proximal fragment. The posterolateral approach was used in several studies as the preferred method for antiglide plating ${ }^{[7,8]}$. The advantage of using posters lateral approach is that the co existing poster lateral malleolus of distal tibia in a SER pattern can also be addressed using same approach.

Peroneal tendinopathy is frequently discussed complication associated with posterior plating. Only one study showed the higher incidence of peroneal tendinitis, while 30 of 70 (43\%) patients had discomfort or signs of peroneal tendinitis ${ }^{[13,14]}$. But, we did not encounter any case of peroneal tendinitis in our study group, although few hardware irritation was observed in cases particularly where an oblique lag screw was inserted. The head of these oblique screw was observed irritating the perineal tendons and that improved immediately following hardware removal. Number of screws in distal fragment can be strategically reduced or avoided in different injury and stability patterns to avoid such irritations due to screw heads ${ }^{[15]}$.

\section{Conclusion}

Antiglide plating for SER pattern injuries is a well described procedure with added advantages of having good biomechanics stability due to its configuration. Advantage of posterolateral approach to distal fibula to insert a posterior antiglide plate benefits to have an additional exposure to posterolateral fragment of tibia in SER injury patterns. We believe that the use of the antiglide plate in a posterolateral position, without using unreasonable distal screws, is an appropriate method of fixation in every short oblique lateral malleolus fractures occurring as a part of SER pattern injury of ankle.

\section{References}

1. Lauge-Hansen N. Fractures of the ankle. II. combined experimental-surgical and experimental-roentgenologic investigations. Arch Surg 1950;60:957-985.

2. Singh R, Kamal T, Roulohamin N, Maoharan G, Ahmed B, Theobald P. Ankle fractures a literature review of current treatment methods. Open J Orthoped. 2014;4:292-303.

3. Ramsey PL, Hamilton W. Changes in tibiotalar area of contact caused by lateral talar shift. J Bone Joint Surg (Am.) 1976;58:356-357.

4. Singh R, Kamal T, Roulohamin N, Maoharan G, Ahmed $\mathrm{B}$, Theobald P. Ankle fractures a literature review of current treatment methods. Open J Orthoped. 2014;4:292-303.

5. Brunner CF, Weber BG, Springer-Verlag; New York: Special Techniques in Internal Fixation 1982.

6. Schaffer JJ, Manoli A. The antiglide plate for Distal Fibular Fixation. A biomechanical comparsion with fixation with a lateral plate. J Bone Joint Surg. 1987;69A:596.

7. Minihane $\mathrm{KP}$, Lee $\mathrm{CH}$, Ahn $\mathrm{CH}$, Zhang LQ, Merk BR. Comparison of lateral locking plate and antiglide plate for fixation of distal fibular fractures in osteoporotic bone: a biomechanical study. J Orthop Trauma 2006;20:526-566

8. Ha WJ, Kim HB, Ko MS, Yoon HK. Comparsion of antiglide plate fixation and lateral plate fixation for Danis-Weber type B isolated malleolar fractures. J Korean Foot Ankle Soc. 2011;15(3):153-158.

9. Velez NM, Moreno AS, Martínez OS, Gutiérrez EJ. 
Posterior antiglide plate vs lateral plate to treat Weber type B ankle fractures. Acta Ortopéd Mexicana. 2004;18(1):39-44.

10. Lamontagne J, Blachut PA, Broekhuyse HM, O’ Brien PJ, Meek RN. Surgical treatment of a displaced lateral malleols Fracture: the antiglide technique versus lateral plate fixation. J Orthop Trauma. 2002;16(7):498-502.

11. Treadwell JR, Fallat LM. The antiglide plate for the Danis-Weber type-B fibular fracture:a review of 71 cases. J Foot Ankle Surg. 1993;32(6):573-579.

12. Wissing JC, van Laarhoven CJHM, van der Werken C. The posterior antiglide plate for fixation of fractures of the lateral malleolus. Injury. 1992;23(2):94-96.

13. Weber M, Krause F. Peroneal tendon lesions caused by antiglide plate sused for fixation of lateral malleolar fractures: the effect of plate and screw position. Foot Ankle Int 2005;26(4):281-285.

14. Ahn J, Kim S, Lee JS, Woo K, Sung KSS. Incidence of peroneal tendinopathy after application of a posterior antiglide plate for repair of supination external rotation lateral malleolar fractures. J Foot Ankle Surg. 2016;55:90-93.

15. Litte MTM, Berkes MB, Lazaro LE, Sculco PK, Helfet DL, Lorich DG. Complications following treatment of supination external rotation ankle fractures through the posterolateral approach. Foot Ankle Int. 2013;34(4):523529. 